\title{
Genetic prerequisites for additive or synergistic actions of 5-fluorocytosine and fluconazole in baker's yeast
}

Correspondence

Friedhelm Meinhardt

meinhar@uni-muenster.de

Received 29 April 2008

Revised 12 June 2008

Accepted 19 June 2008

\author{
John P. Paluszynski, Roland Klassen and Friedhelm Meinhardt
}

Institut für Molekulare Mikrobiologie und Biotechnologie, Westfälische Wilhelms-Universität Münster, Corrensstr. 3, D-48149 Münster, Germany

\begin{abstract}
During applications of 5-fluorocytosine (5FC) and fluconazole (FLC), additive or synergistic action may even occur when primary resistance to $5 \mathrm{FC}$ is established. Here, we analysed conjoint drug action in Saccharomyces cerevisiae strains deficient in genes known to be essential for 5FC or FLC function. Despite clear primary resistance, residual 5FC activity and additive 5FC+FLC action in cells lacking cytosine permease (Fcy2p) or uracil phosphoribosyl transferase (Fur1p) were detected. In contrast, $\Delta f c y 1$ mutants, lacking cytosine deaminase, became entirely resistant to $5 \mathrm{FC}$, concomitantly losing $5 \mathrm{FC}+\mathrm{FLC}$ additivity. Disruption of the orotate phosphoribosyltransferase gene (URA5) in the wild-type led to low-level 5FC tolerance, while an alternative orotate phosphoribosyltransferase, encoded by URA10, contributed to $5 \mathrm{FC}$ toxicity only in the $\Delta u r a 5$ background. Remarkably, combination of $\Delta u r a 5$ and $\Delta f u r 1$ resulted in complete 5FC resistance. Thus, yeast orotate phosphoribosyltransferases are involved in 5FC metabolism. Similarly, disruption of the ergosterol $\Delta^{5,6}$-desaturase-encoding gene ERG3 resulted only in partial resistance to FLC, and concomitantly a synergistic effect with 5FC became evident. Full resistance to FLC occurred in $\Delta$ erg $3 \Delta$ erg 11 double mutants and, simultaneously, synergism or even an additive effect with FLC and 5FC was no longer discernible. Since the majority of spontaneously occurring resistant yeast clones displayed residual sensitivity to either 5FC or FLC and those strains responded to combined drug treatment in a predictable manner, careful resistance profiling based on the findings reported here may help to address yeast infections by combined application of antimycotic compounds.
\end{abstract}

\section{INTRODUCTION}

The fluorinated pyrimidine 5-fluorocytosine (5FC) is one of the most widely used antimycotic agents, capable of disrupting both DNA and protein synthesis in fungal cells (Ghannoum \& Rice, 1999). Fluconazole (FLC) on the other hand, exhibits a completely different mode of action, targeting Erg11, essential for the production of ergosterol in fungi (Kalb et al., 1987; Vanden Bossche, 1985). These two agents are routinely administered in combination to combat a broad spectrum of fungal infections (Mukherjee et al., 2005; Johnson et al., 2004). The desired effect of such combination therapy is an additive or synergistic increase in antifungal activity when compared to singly applied compounds. In a previous study, Saccharomyces cerevisiae was used as a model organism to identify putative permeases which play a role in 5FC toxicity. In S. cerevisiae, 5FC uptake is brought about mainly by the cytosine permease Fcy2; however, in its absence, several other permeases ensure residual 5FC influx (Paluszynski et al.,

Abbreviations: 5FC, 5-fluorocytosine; 5FU, 5-fluorouracil; FIC, fractional inhibitory concentration; FLC, fluconazole.
2006). However, the impact of disruptions in the 5FC metabolic pathway on synergy when combined with FLC has yet to be studied. Interestingly, in Cryptococcus neoformans, synergism was observed in cases in which primary resistance to one of the agents occurred (Schwarz et al., 2003, 2006, 2007), but the reasons remained unknown. Depending on the agent and yeast species, however, adverse (antagonistic) effects of a combination therapy are also documented (Te Dorsthorst et al., 2002). The outcome of a combined antimycotic treatment apparently varies within genera, species and even isolates, and is thus hardly predictable. Phylogenetic analyses of pathogenic yeast species indicated that $S$. cerevisiae is closely related to the major opportunistic fungal pathogen Candida albicans (Barns et al., 1991; Hendricks et al., 1989; Lupetti et al., 2002). Thus, despite the above constraints, $S$. cerevisiae may serve as a valuable model to study antifungal drug action (Agarwal et al., 2003).

5FC is a prodrug, which to exert its action requires uptake and metabolism to either 5-fluorouridine triphosphate (5FUTP, formed from 5FUDP; see Fig. 1) or 5-fluorodeoxyuridine monophosphate (5FdUMP), the former directly 


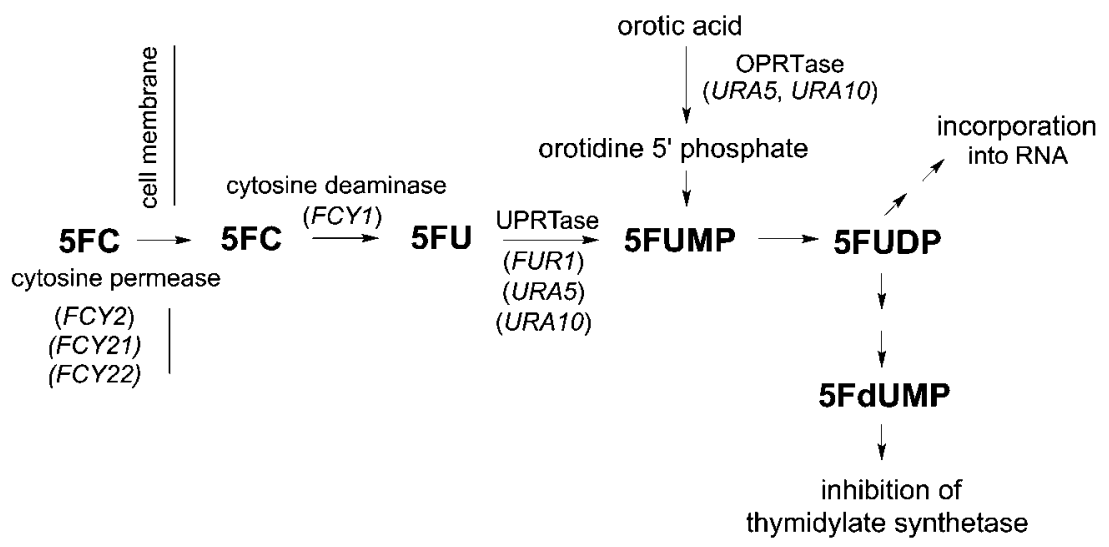

Fig. 1. Metabolism of 5-fluorocytosine in fungi. Enzyme and substrate names are abbreviated as follows: OPRTase, orotate phosphoribosyltransferase; 5FC, 5-fluorocytosine; 5FU, 5-fluorouracil; UPRTase, uridine phophoribosyltransferase; 5FUMP, 5-fluorouridine monophosphate; 5-FUDP, 5-fluorouridine diphosphate; 5FdUMP, fluorodeoxyuridine monophosphate.

disturbing transcription and the latter - via inhibition of thymidylate synthetase and subsequent dTTP depletion aborting DNA synthesis (Polak \& Scholer, 1975; Hartmann \& Heidelberger, 1961; Whelan, 1987; Wadler et al., 1998). Essential steps in intracellular 5FC metabolism are the conversion to 5-fluorouracil (5FU) by the cytosine deaminase Fcyl and subsequent processing to 5-fluorouridine monophosphate by the uracil phosphoribosyltransferase Fur1 (Chevallier et al., 1975; Vanden Bossche et al., 1994, 1987; Kern et al., 1990; Kurtz et al., 1999).

In S. cerevisiae, as for C. albicans and Candida glabrata, mutations in any of the corresponding genes involved in prodrug uptake and metabolism result in 5FC tolerance (Fasoli et al., 1990), explaining the known rapid establishment of spontaneous resistance (Alexander \& Perfect, 1997; Vanden Bossche et al., 1987; Paluszynski et al., 2006).

FLC and several other azole antimycotics interfere with the biosynthesis of ergosterol, a fungal-specific sterol that is important for membrane integrity (Smith et al., 1996, Bammert \& Fostel, 2000). The specific target of antimycotic azoles is the lanosterol $14 \alpha$-demethylase, encoded by the ERG11 gene (Kalb et al., 1987; Vanden Bossche, 1985). By binding the iron atom of the haem moiety, activation of oxygen, necessary for demethylation of lanosterol, is prevented (Joseph-Horne \& Hollomon, 1997), eventually resulting in the accumulation of toxic $14 \alpha$-methylergosta-

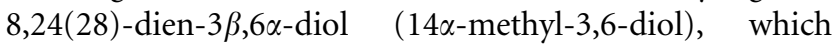
impairs membrane function (Kelly et al., 1995).

Mechanisms of azole resistance in S. cerevisiae and $C$. albicans include overproduction or alteration of Erg11p, or modification of downstream enzymes (Bard et al., 1993; Vanden Bossche et al., 1992; Hitchcock, 1991). The loss of function of the sterol $\Delta^{5,6}$-desaturase encoded by ERG3 results in accumulation of episterol, which permits fungal growth in the presence of azole drugs (Arthington et al., 1991; Watson et al., 1988; Kelly et al., 1995). However, disruption of the aforementioned ERG11 gene was shown to be lethal in S. cerevisiae, as accumulation of $14 \alpha$-methyl3,6-diols facilitates growth only under anaerobic conditions and in ergosterol-supplemented media (Watson et al.,
1989; Bard et al., 1993). This growth arrest can be circumvented by the inactivation of $E R G 3$, which results in the accumulation of methylfecosterol instead of $14 \alpha-$ methyl-3,6-diol. In contrast, C. albicans ERG11 null mutants are capable of survival in the absence of a suppressor mutation in $E R G 3$, albeit with a severe growth defect (Sanglard et al., 2003). Hence, C. albicans either produces different levels of the diol and/or is less sensitive to its lethal effects as compared to S. cerevisiae (Watson et al., 1989; Bard et al., 1993).

To determine the prerequisites underlying the synergistic or additive increase in antifungal action of $5 \mathrm{FC} / \mathrm{FLC}$ combinations, we set up a $S$. cerevisiae model system consisting of an isogenic strain collection with combinations of defined deletions in genes important for 5FC and/ or FLC toxicity. Sensitivity profiling revealed residual drug responses and additive action of the two drugs in the majority of strains despite the establishment of primary resistance. The genetic basis of such residual activity was investigated and identified as being essential for increased drug action in combined applications of 5FC and FLC.

\section{METHODS}

Strains, plasmids and growth conditions. Strains and plasmids used in this study are listed in Table 1. Cultivation was performed in complete YPD (1\%, w/v, Bacto-yeast extract, $2 \%$, w/v, Bactopeptone, $2.2 \%$ w w, glucose) or YNB (Yeast Nitrogen Base, Difco) media supplemented with (L-leucine $10 \mu \mathrm{g} \mathrm{ml}^{-1}$, L-methionine $10 \mu \mathrm{g}$ $\mathrm{ml}^{-1}$, uracil $200 \mu \mathrm{g} \mathrm{ml}{ }^{-1}$, uridine $100 \mu \mathrm{g} \mathrm{ml}^{-1}$ ) when required. Media were prepared according to Kaiser et al. (1994). Strains were cultivated at $30{ }^{\circ} \mathrm{C}$ in YPD liquid or on agar medium. Escherichia coli strains were grown in $\mathrm{LB}(1 \%$, w/v, peptone, $0.5 \%$, w/v, yeast extract, $0.5 \%, \mathrm{w} / \mathrm{v}, \mathrm{NaCl}, \mathrm{pH} 7.3$ ) at $37{ }^{\circ} \mathrm{C}$ (Sambrook et al., 1989) with a working concentration of ampicillin of $100 \mu \mathrm{g} \mathrm{ml}^{-1}$.

DNA techniques. Chromosomal yeast DNA was isolated from $50 \mathrm{ml}$ YPD cultures essentially as described by Kaiser et al. (1994). Restriction and ligation of DNA was carried out according to the suppliers' recommendations (NEB and MBI-Fermentas). For Southern analyses (Southern, 1975) DNA fragments were labelled by applying the DIG-DNA-labelling and detection kit from Roche Biochemicals. 
Table 1. Strains and plasmids used in this study

\begin{tabular}{|c|c|c|}
\hline Strain or plasmid & Genotype & Source \\
\hline \multicolumn{3}{|l|}{ S. cerevisiae } \\
\hline BY4741 & MATa his 3 leu 2 met15 ura3 & EUROSCARF \\
\hline CG379 & MAT $\alpha$ ade5-1 leu2-2 trp1-289 ura3-52 his7-2 & Bard et al. (1993) \\
\hline BY $\Delta f c y 1$ & As for BY4741, additionally $f_{c} y 1$ & EUROSCARF \\
\hline BY $\Delta f c y 2$ & As for BY4741, additionally $f_{c y 2}$ & EUROSCARF \\
\hline BY $\Delta u r a 5$ & As for BY4741, additionally ura5:: SpHIS5 & This work \\
\hline BY $\Delta$ ura 10 & As for BY4741, additionally ura $10::$ KlLEU2 & This work \\
\hline BY $\Delta$ ura5 $\Delta$ ura 10 & As for BY474,1 additionally ura5:: SpHIS5 ura10:: KlLEU2 & This work \\
\hline BY $\Delta$ furl & As for BY4741, additionally fur $1::$ KlURA3 & This work \\
\hline BY $\Delta$ furl $\Delta$ ura5 & As for BY4741, additionally fur $1:$ KlURA3 ura $5:$ SpHIS5 & This work \\
\hline BY $\Delta$ erg3 & As for BY4741, additionally erg3:: KlLEU2 & This work \\
\hline BY $\Delta \operatorname{erg} 3 \Delta \operatorname{erg} 11$ & As for CG379, additionally $\operatorname{erg} 3:: K l L E U 2 \operatorname{erg} 11:: K l U R A 3$ & Bard et al. (1993) \\
\hline BY $\Delta f_{c y 1} \Delta \operatorname{erg} 3$ & As for BY4741, additionally fcyl erg3:: KlLEU2 & This work \\
\hline BY $\Delta f c y 2 \Delta \operatorname{erg} 3$ & As for BY4741, additionally $f c y 2 \operatorname{erg} 3:: K l L E U 2$ & This work \\
\hline BY $\Delta$ fur1 $\Delta \operatorname{erg} 3$ & As for BY4741, additionally fur $1::$ KlURA3 $\operatorname{erg} 3::$ KlLEU2 & This work \\
\hline \multicolumn{3}{|l|}{ E. coli } \\
\hline JM109 & $\mathrm{F}^{\prime}$ traD36 $\operatorname{proA}^{+} B^{+}$lacI $^{q} \Delta($ lacZ $) \mathrm{M} 15 / \Delta($ lac-pro $A B) \operatorname{gln} V$ thi & Yanisch-Perron et al. (1985) \\
\hline \multicolumn{3}{|l|}{ Plasmids } \\
\hline $\mathrm{pSK}-$ & ColE1 ori, $\mathrm{Amp}^{\mathrm{R}}$ lac $Z$ & Stratagene \\
\hline pUG72 (AF298788) & loxP-KlURA3-loxP, $\mathrm{Amp}^{\mathrm{R}}$ & EUROSCARF \\
\hline pUG73 (AF298792) & loxP-KlLEU2-loxP, $\mathrm{Amp}^{\mathrm{R}}$ & EUROSCARF \\
\hline
\end{tabular}

Transformation and gene disruptions. E. coli JM109 was transformed by the $\mathrm{CaCl}_{2}$ method as described by Sambrook et al. (1989). Transformants of $S$. cerevisiae were obtained according to Gietz \& Schiestl (1995), and selected on YNB agar.

The ERG3 gene was disrupted using the KlLEU2 marker gene cloned in pUG73. ERG3 was amplified and blunt-end inserted into the HincII site of the pSK plasmid vector (see Table 2 for primers). Subsequently, the KLLEU2 gene from pUG73 was excised (HincII and PvuII) and inserted into the internal ERG3 HincII site. The disruption cassette erg3: : LEU2 was introduced into $S$. cerevisiae BY4741, S. cerevisiae $\Delta f_{c y 1}$ and $S$. cerevisiae $\Delta f c y 2$ strains (Table 1). Mutants obtained from EUROSCARF (Frankfurt, Germany), $\Delta f c y 1$ and $\Delta f c y 2$, were verified using primer pairs Fcy1F/R and Fcy2F/R, respectively. Homologous recombination with the ERG3 disruption cassette was checked by PCR, in a MiniCycler (MJ Research Biozym) and Southern analysis.

The disruption of URA5, URA10 and FUR1 was carried out essentially as described previously (Wach et al., 1994; Gueldener et al., 2002). For URA5 disruption, primers (URA5koF and URA5koR) flanking the SpHIS5 marker gene from pUG27 and an additional 45 bp homologous to URA5 were used. URA10 was disrupted using the KlLEU2 marker gene from pUG73, with primers URA10koF and URA10koR. FUR1 was disrupted using FurlkoF and FurlkoR primers flanking the KlURA3 marker gene. The URA5:: SpHIS5 knockout cassette was transformed into S. cerevisiae $\Delta$ fur 1 and $\Delta$ ura 10 , resulting in $\Delta u r a 5 \Delta$ fur 1 and $\Delta u r a 5$ $\Delta$ ura 10 double mutant strains. Disruption of URA5 was PCR verified, employing primer pairs URA5outR/HIS5up and URA5outF/HIS5down, respectively. For verification of URA10 and FUR1 knockouts, primer pairs LEU2down/URA10outF, LEU2up/URA10outR and URA3down/ FurloutF, URA3up/FurloutR were applied.

In vitro drug susceptibility tests. Stock solutions of 5 FC and FLC obtained from MP Bio-medicals, Frankfurt, Germany, were established with a final concentration of $5 \mathrm{mg} \mathrm{ml}^{-1}$, and after sterilefiltration (cellulose acetate membrane, pore size $0.2 \mu \mathrm{m}$; Millipore), stored at $-20{ }^{\circ} \mathrm{C}$. Prior to use drugs were added to YPD agar at approximately $50{ }^{\circ} \mathrm{C}$ to establish appropriate concentrations. For testing resistance rapidly, cultures were diluted $10^{-1}$ to $10^{4}$ and $8 \mu \mathrm{l}$ of each dilution was spotted onto the agar plates. For proper quantification, $200 \mu \mathrm{l}$ volumes of liquid YPD medium containing 5FC, FLC, and both in combination, were inoculated with $1 \times 10^{6}$ yeast cells and incubated at $30{ }^{\circ} \mathrm{C}$ in U-profile 96-well microtitre plates (Carl Roth) for $24 \mathrm{~h}$; growth was monitored spectroscopically at $600 \mathrm{~nm}$ as previously described (Paluszynski et al., 2006).

Where applicable, the minimal inhibitory concentration (MIC) for 5FC and FLC was read as the lowest drug concentration that gave $50 \%$ or more growth inhibition, which is defined as MIC-2 as described by the National Committee for Clinical Laboratory Standards (NCCLS, 1998). The fractional inhibitory concentration (FIC) was calculated to quantify drug interaction (Elfopouios \& Moellering, 1991), being defined as synergistic if the FIC was $\leqslant 0.5$, additive if FIC was $>0.5$ and $\leqslant 1$, indifferent if $1<$ FIC $\leqslant 4$, and antagonistic if FIC was $>4$.

The FIC index was determined as follows: $\mathrm{FIC}=[(\mathrm{MIC}$ of drug $\mathrm{A}$, in combination $) /($ MIC of drug $\mathrm{A}$, tested alone $)]+[$ (MIC of drug $\mathrm{B}$, in combination $) /($ MIC of drug B, tested alone)].

Measurement of growth of $\Delta$ erg 3 and $\Delta$ erg 3 serg 11 mutants. To measure the growth of $\Delta$ erg 3 and $\Delta \operatorname{erg} 3 \Delta \operatorname{erg} 11$ mutant strains, in comparison to the wild-type, growth curves were established by inoculating $1 \%$ of overnight pre-cultures in $50 \mathrm{ml}$ YPD medium. Cultivation was performed in the presence and absence of FLC at $30{ }^{\circ} \mathrm{C}$, with the final concentration of FLC being $1 \mathrm{mg} \mathrm{ml}^{-1}$. Growth was monitored spectroscopically at $600 \mathrm{~nm}$ until strains reached the stationary phase.

\section{RESULTS AND DISCUSSION}

\section{Conjoint actions of 5FC and FLC in defined S. cerevisiae mutants}

For systematic analysis of conjoint antifungal activities of 5FC and FLC in resistant yeast strains, we assembled a set 
Table 2. Oligonucleotides used for PCR along with target sequences and positions according to the Saccharomyces genome database (http://www.yeastgenome.org/)

\begin{tabular}{|c|c|c|c|}
\hline Primer & Sequence & Length (bp) & $\begin{array}{l}\text { Target sequence (locus } \\
\text { and nucleotide positions) }\end{array}$ \\
\hline LEU2up & GGCGTATAGACCCAATTCC & 19 & LEU2 nt 1041-1023 \\
\hline Erg3F & ATGGATTTGGTCTTAGAAGTCGC & 23 & ERG3 Chrom. XII nt 5001-5023 \\
\hline Erg3R & TCAGTTGTTCTTCTTGGTATTTGGG & 25 & ERG3 Chrom. XII nt 6074-6098 \\
\hline Erg3out5 & GGTTGCAGAGGAGGTCAG & 18 & ERG3 Chrom. XII nt 4928-4945 \\
\hline FcylF & GGGTATGGACATTGCCTATG & 20 & FCY1 Chrom. XVI nt 677198-677217 \\
\hline FcylR & CCAATCCTGAGGTCTTTCATCG & 22 & FCY1 Chrom. XVI nt 677595-677616 \\
\hline Fcy2F & CGTTGAATCATCAGAGGCCAC & 21 & FCY2 Chrom. V nt 266655-266675 \\
\hline Fcy2R & GGTGCCCACAAAGCTTGAGCG & 21 & FCY2 Chrom. V nt 267663-267683 \\
\hline URA3up & GACGCTGGCGTACTGGC & 17 & $U R A 3$ nt $1067-1051$ \\
\hline URA3down & GCCAGTACGCCAGCGTC & 17 & URA3 nt 1067-1051 \\
\hline FurlkoR & $\begin{array}{l}\text { GTATCTGTCACCAAAGTCACCCAACCCTGGAACTAGATACTTG- } \\
\text { G/CATAGGCCACTAGTGGATCTG }\end{array}$ & 65 & $\begin{array}{l}\text { FUR1/URA3 nt 362714-362757/1604- } \\
1583\end{array}$ \\
\hline FurloutF & GGCCGGTTTTTCTATAAGC & 19 & FUR1 Chrom. VIII nt 361837-361819 \\
\hline FurloutR & GGCTAGAGGACAGTACCCG & 19 & FUR1 Chrom. VIII nt 363096-363077 \\
\hline URA5koF & $\begin{array}{l}\text { CGAATGCCAGGCTCTAAGATTTGGTTCATTCAAGTTGAAATCA- } \\
\text { G/CAGCTGAAGCTTCGTACGC }\end{array}$ & 63 & $\begin{array}{l}\text { URA5/HIS5 Chrom. XIII nt 56773- } \\
\text { 57453/1089-1073 }\end{array}$ \\
\hline URA5koR & $\begin{array}{l}\text { CTGTTATTCTGCCTTCCAAATAGGTAATTATGTGAATTAAGGAG- } \\
\text { AC/GCATAGGCCACTAGTGGATCTG }\end{array}$ & 68 & $\begin{array}{l}\text { URA5/HIS5 Chrom. XIII nt 57406- } \\
\text { 57342/1089-1073 }\end{array}$ \\
\hline URA5outF & GTAACTGCCCTACTTTCC & 18 & URA5 Chrom. XIII nt 56272-56289 \\
\hline URA5outR & GGCAAGAGGTACCGAAAC & 18 & URA5 Chrom. XIII nt 57491-57509 \\
\hline URA10koF & $\begin{array}{l}\text { TGAACTGGGATTAGAATGCAAAGCACTAAGATTTGGGTCATTC- } \\
\text { AAG/CAGCTGAAGCTTCGTACGC }\end{array}$ & 65 & $\begin{array}{l}\text { URA10/LEU2 Chrom. XIII nt 807595- } \\
807640 / 1051-1067\end{array}$ \\
\hline
\end{tabular}

of isogenic derivatives of $S$. cerevisiae BY4741 carrying mutations expected to provoke 5FC or FLC resistance. 5FC resistance was achieved by disruption of FCY1, FCY2 and FUR1, encoding cytosine deaminase, purine-cytosine permease and uracil phosphoribosyltransferase, respectively (see also Fig. 1); FLC resistance was achieved by disruption of ERG3, encoding the C-5 sterol desaturase.

Resistance levels of respective mutants of the BY4741 background were initially monitored using a drop dilution plate assay employing various concentrations of $5 \mathrm{FC}$ and FLC. As to be expected, $\Delta f c y 1, \Delta f c y 2$ and $\Delta f u r 1$ mutations clearly conferred 5FC resistance (Fig. 2a), whereas FLC resistance was solely observed in the $\Delta \operatorname{erg} 3$ strain (Fig. 2b). There was no cross-resistance to FLC in $\Delta f c y 1, \Delta f c y 2$ or $\Delta$ furl strains and vice versa; as for the wild-type $5 \mathrm{FC}$ sensitivity was seen in the $\Delta \operatorname{erg} 3$ mutant.

Growth capabilities in the presence of a broad concentration range of both 5FC and FLC, singly and in combination, were scored with the microtitre plate assay, which revealed differential effects of individual gene disruptions on the efficiency of combined drug treatments (Fig. 3). Neither in wild-type nor in any of the mutant strains were antagonistic effects of combined 5FC/FLC application observed. Rather, clear additive interactions of both drugs were detected in the wild-type $(\mathrm{FIC}=0.74)$ and the $\Delta f c y 2$ mutant $(\mathrm{FIC}=0.576)$ or synergism in the case of the $\Delta \operatorname{erg} 3$ mutant $(\mathrm{FIC}=0.20)$, despite the fact that the latter two exhibit resistance to 5FC or FLC (Figs 3 and 4a). Such an increase in efficiency for $5 F C+$ FLC was also seen in the $\Delta$ furl mutant $(\mathrm{FIC}=0.63)$ (Fig. 3d). Significantly, there is a remarkable difference in the residual responses to the singly applied antimycotics that correlates with additive or synergistic interactions with the second drug. Despite the response being clearly distinct in strains deficient in either FCY2 or FUR1, both show clear growth inhibition by $5 \mathrm{FC}$, dose-dependent in $\Delta f_{c} y 2$ and dose-independent in the $\Delta$ fur1 mutant (Fig. 3). Similarly, there is an apparent FLCdependent growth retardation in the $\Delta \operatorname{erg} 3$ strain, which is, however, not aggravated with increasing FLC concentrations 
(a)
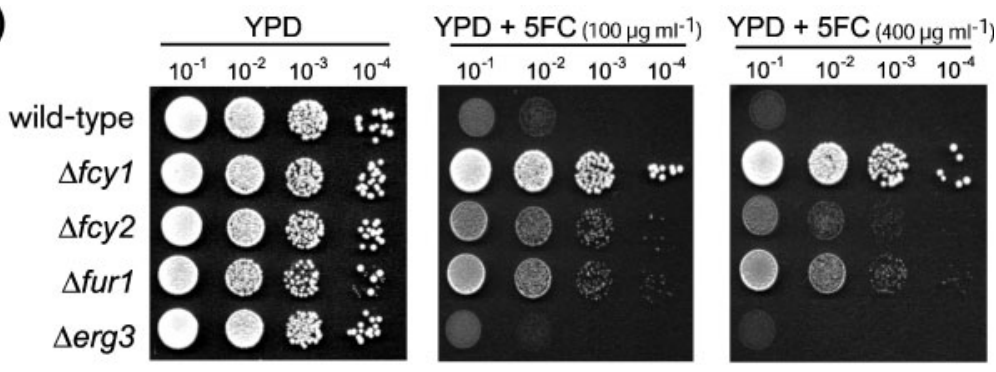

(b)

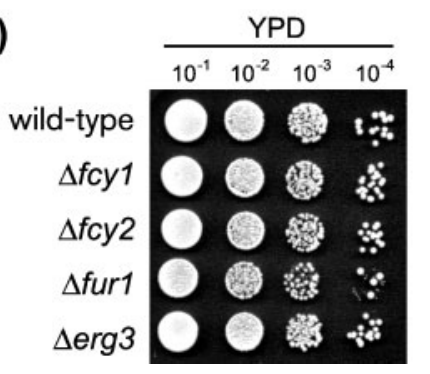

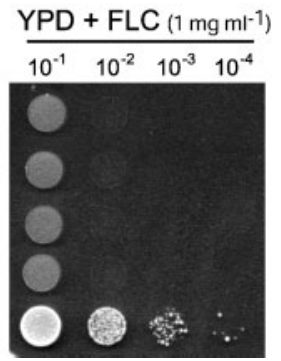

Fig. 2. Drop dilution assay for evaluating the sensitivity of various deletion mutants in $S$. cerevisiae: comparison of $\Delta f c y 1, \Delta f c y 2, \Delta f u r 1$ and $\Delta$ erg 3 mutant strains to the wild-type BY4741 when treated with (a) 5FC and (b) FLC. Each row represents a serial dilution from $10^{-1}$ to $10^{-4}$ from left to right; $8 \mu$ of each dilution was spotted onto each series of YPD plates. The concentrations of $5 \mathrm{FC}$ and FLC are given above each panel. Without addition of antimycotics (YPD) all samples displayed the same growth capabilities and very similar cell numbers. Resistant phenotypes can be directly monitored by growth.
(Fig. 4a). By comparing growth of wild-type and $\Delta$ erg 3 cells, a general growth-affecting defect in $\Delta$ erg3 became obvious; however with FLC, growth was additionally retarded (Fig. $4 \mathrm{~b})$. Thus, FLC inhibits growth even in cells carrying the FLC resistance mutation $\Delta$ erg3.

Mutation of ERG3, a late ergosterol biosynthesis gene, results in altered membrane sterol composition, with ergosterol being replaced by episterol (Arthington et al., 1991). The Erg3 FLC-toxicity-promoting reaction is the generation of a toxic diol from $14 \alpha$-methylfecosterol, the latter being formed from lanosterol by inhibition of the 14$\alpha$ demethylase (Erg11) by FLC (Bard et al., 1993; Watson et al., 1989). Thus, $\Delta$ erg3 mutants tolerate FLC, as no toxic diols are synthesized, but concomitantly exhibit an altered membrane sterol composition probably accounting for the observed slow growth of these mutants (see also Fig. 4).

Although the main target of FLC, Erg11, is normally essential, it is dispensable when Erg3 is additionally knocked out, as this abrogates formation of toxic diols (Bard et al., 1993; Watson et al., 1989). $\Delta$ erg3 $\Delta$ erg11 cells displayed full FLC resistance, but again, the strong FLC-independent growth defect became obvious (Fig. 4a, c). Interestingly, growth of the double mutant $(\Delta \operatorname{erg} 3 \Delta \operatorname{erg} 11)$, irrespective of the presence or absence of FLC, is comparable to that of the $\Delta$ erg3 strain with FLC. In each of the latter, the ergosterol biosynthesis pathway yields $14 \alpha$-methylfecosterol, evidently causing a more pronounced growth impairment than episterol but less than $14 \alpha$-methyl-3,6-diol formed in wildtype cells when exposed to FLC (Fig. 4d). Importantly, synergistic actions of FLC and 5FC, as seen in $\Delta$ erg 3 cells, are cancelled by the additional $\Delta$ erg 11 mutation, indicating the requirement of at least residual FLC-mediated growth retardation for synergistic or additive drug action.

As for the $\Delta \operatorname{erg} 3 \Delta \operatorname{erg} 11$ mutant, the additive response was abolished when FCY1 was lacking, with both displaying an
FIC of 1 and, at least consistent with residual sensitivity being crucial for additive action, $\Delta f c y 1$ mutants displayed no response to 5FC regardless of the concentration applied (Fig. 3b). Thus, synergistic or additive antifungal action of 5FC/FLC treatment is seen in $\Delta e r g 3, \Delta f c y 2$ and $\Delta f u r 1$ strains, whereas such an effect is absent in $\Delta \operatorname{erg} 3 \Delta \operatorname{erg} 11$ double and $\Delta f c y 1$ single mutants, which do not respond to either FLC or 5FC, respectively.

\section{FC response in fur1 mutants}

We have recently shown that toxicity of $5 \mathrm{FC}$ in $\Delta f c y 2$ mutants is due to the presence of several other permeases capable of low-level 5FC transport in $S$. cerevisiae (Paluszynski et al., 2006). Growth inhibition of $\Delta$ fur1 mutants by 5FC must, however, occur in a different manner, since the Furl-catalysed conversion of 5FU to 5FUMP is crucial for downstream effects of 5FC (Fig. 1). Unlike mammals, S. cerevisiae lacks alternative enzymes capable of 5FU metabolism, such as thymidine or uridine phosphorylase (Jund \& Lacroute, 1970). According to the Candida genome database (http://www.candidagenome. org/) and Cryptococcus neoformans genome project (http:// www.tigr.org/tdb/e $2 \mathrm{k} 1 / \mathrm{cna} 1 /$ ), there is also no evidence for the presence of thymidine or uridine phosphorylases in these genera. In mammals, 5FU can also be metabolized by orotate phosphoribosyltransferase, an enzyme involved in de novo pyrimidine biosynthesis (Peters et al., 1984); the engagement of the respective yeast enzymes in 5FC antimycotic activity has, however, yet to be investigated. S. cerevisiae possesses two homologous orotate phosphoribosyltransferases, Ura5 and Ura10 (Fig. 5a), which are only distantly related to the functional analogues of mammals (de Montigny et al., 1990). Hence, the involvement of such yeast orotate phosphoribosyltransferases in 5FU metabolism was checked by disrupting 

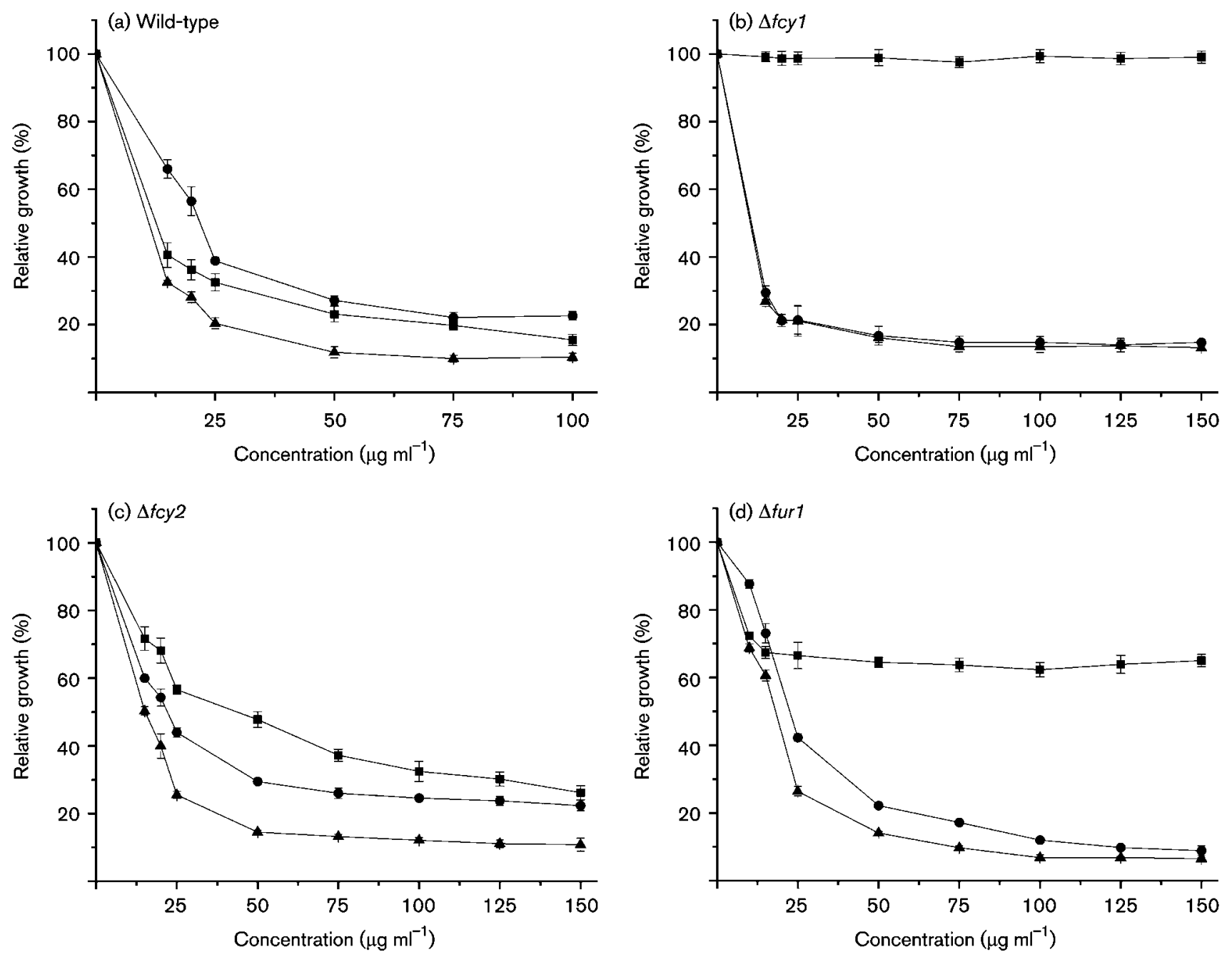

Fig. 3. Microtitre plate assay to monitor the response of yeast strains carrying single mutations in the cytosine metabolic pathway to $5 \mathrm{FC}(\boldsymbol{\square}), \mathrm{FLC}(\boldsymbol{\bullet})$ and $5 \mathrm{FC}+\mathrm{FLC}(\boldsymbol{\Delta})$. (a) S. cerevisiae wild-type BY4741, (b) $\Delta f c y 1$, (c) $\Delta f c y 2$, (d) $\Delta f u r 1$. Growth was measured as $\mathrm{OD}_{600}$ and is expressed as a percentage relative to the control. Values given represent the mean of at least three experiments, each carried out in triplicate; error bars represent SD (not plotted where smaller than symbols). There is a clear additive effect for $5 \mathrm{FC}$ and FLC for the wild-type (a), for $\Delta f c y 2$ (c) and for $\Delta$ fur 1 (d), whereas $\Delta f c y 1$ (b) behaves differently.

URA5 and URA10 singly and in combination. Effects on 5FC tolerance were subsequently recorded by the microtitre plate assay (Fig. 5b). Indeed, the $\Delta$ ura 5 single mutant displayed moderate resistance at low 5FC concentrations (Fig. 5b). However, $\Delta$ ura10 alone did not affect 5FC tolerance significantly, but slightly contributed to resistance in the $\Delta$ ura 5 background (not shown). Most remarkably, however, full dose-independent 5FC resistance was established when URA5 was disrupted in the $\triangle$ furl strain (Fig. 5b). Thus, both Ura5 and Ura10 are capable of 5FU metabolism and probably mediate residual 5FC toxicity in the absence of Fur1. As homologues of S. cerevisiae Ura5/10 have been identified in a variety of ascomycetous yeast species, including Candida albicans and C. glabrata, and also in the basidiomycetous yeast Cryptococcus neoformans (Fig. 5a), it appears probable that a requirement for uracil phosphoribosyltransferase in 5FC prodrug activation and antifungal activity can generally be bypassed to some extent by orotate phosphoribosyltransferases. Among three loci known to be involved in 5FC uptake and activation (FCY1, FCY2 and FUR1) in S. cerevisiae, only one proved to be essential ( $F C Y 1)$. It has previously been shown that $S$. cerevisiae $\Delta f c y 1$ mutants entirely lack cytosine deaminase activity, and $\Delta f c y 1 \Delta u r a 3$ or $\Delta u r a 2$ double mutants, which are additionally defective in de novo pyrimidine synthesis, are unable to grow with exogenously supplied cytosine (Jund \& Lacroute, 1970; Erbs et al., 1997), clearly excluding an alternative enzyme for bypassing the Fcyl deamination of cytosine or 5FC. In contrast, Fcy2 and Fur1 reactions can be catalysed by structurally or functionally related proteins, thereby explaining successful application of 5FC in instances where (partial) resistance was established by targeted gene disruption in the model system, or by spontaneous mutation in resistant clinical isolates. 
(a) $\Delta$ erg3

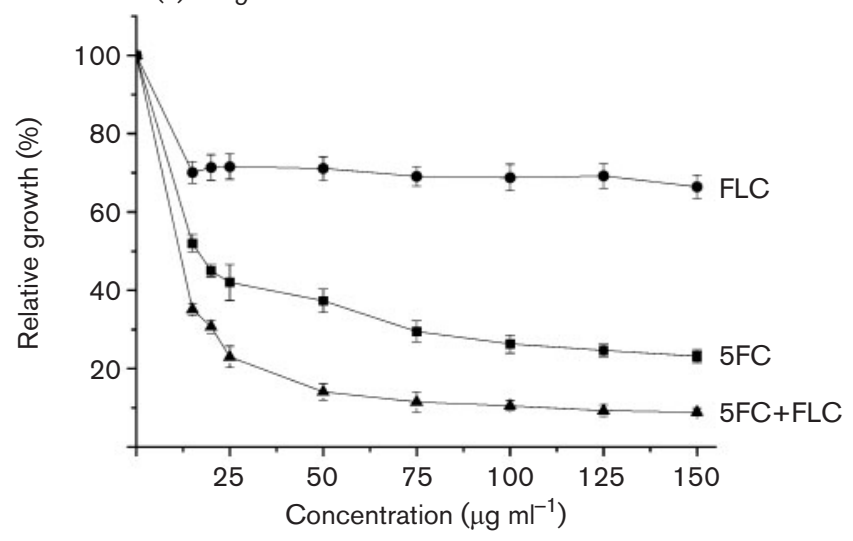

(c) $\operatorname{serg} 3 \operatorname{serg} 11$

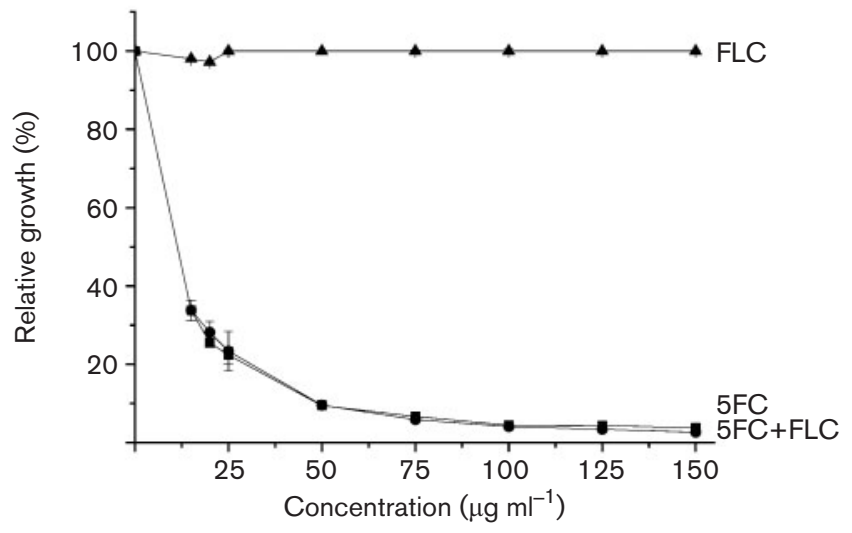

(b)
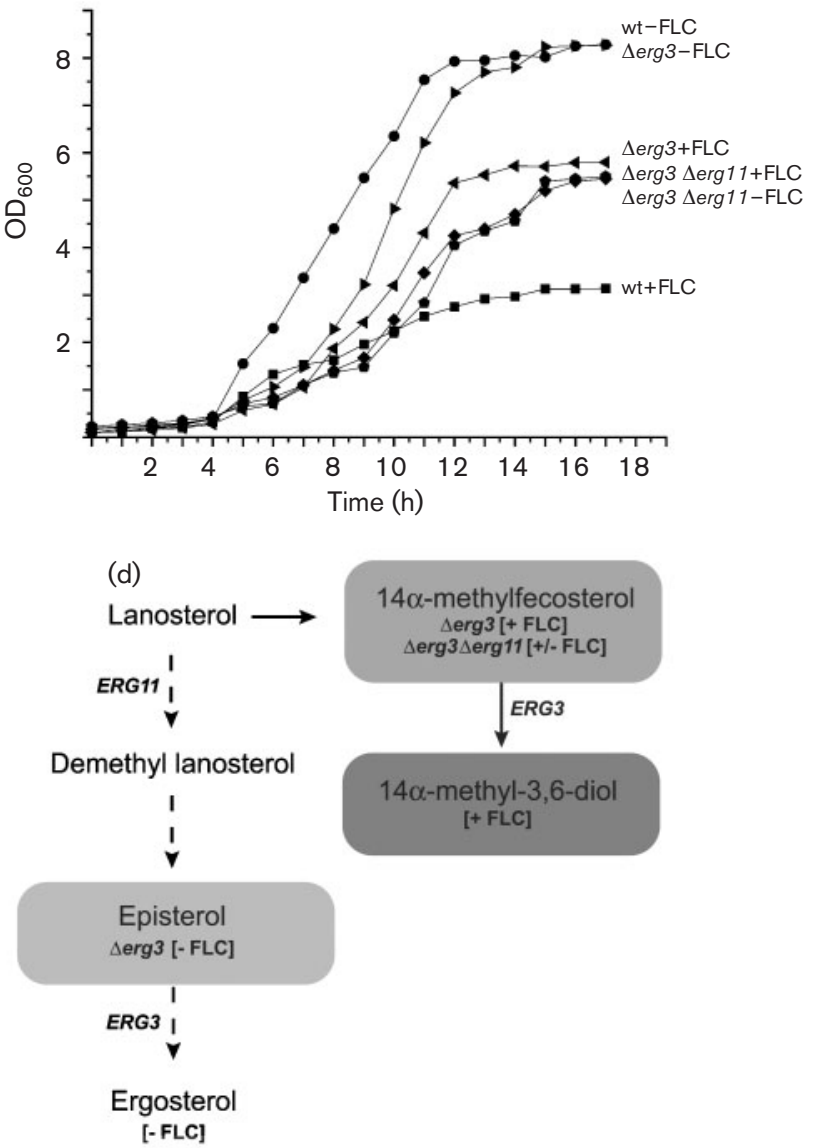

Fig. 4. Assessment of ergosterol mutant strains for FLC resistance using the microtitre plate test and evaluation of growth and synergism in combination with 5FC. (a) Response of $\Delta$ erg3 to FLC (0), 5FC ( $\boldsymbol{\square})$ and FLC $+5 F C(\boldsymbol{\Delta})$. (b) Growth of $\Delta$ erg3, and a $\Delta$ erg3 $\Delta$ erg 11 double mutant, in comparison to wild-type in the presence and absence of FLC. (c) Response of an $\Delta$ erg 3 $\Delta$ erg 11 double mutant to $\operatorname{FLC}(\boldsymbol{\Delta}), 5 \mathrm{FC}(\boldsymbol{\square})$ and $\mathrm{FLC}+5 \mathrm{FC}(\boldsymbol{\bullet})$. (d) Overview of the sterol composition in $\Delta$ erg 3 and $\Delta$ erg 3 $\Delta$ erg 11 mutant strains either treated or not treated with FLC. Error bars represent SD (not plotted where smaller than symbols).

Importantly, clinical isolates of $C$. neofomans displaying resistance to $5 \mathrm{FC}$ but increased susceptibility to $5 \mathrm{FC}$ plus amphotericin B (compared to amphotericin B alone) were shown by complementation to be defective in cytosine permease. It was speculated that addition of amphotericin $\mathrm{B}$, which directly induces membrane damage (de Kruijff et al., 1974; de Kruijff \& Demel, 1974) might restore 5FC penetration, thus explaining synergistic antifungal activity in a cytosine-permease-defective strain (Schwarz et al., 2007). However, synergism was also observed in 5FCresistant isolates when this agent was combined with FLC, which does not directly affect membrane integrity (Allendoerfer et al., 1991). As we detected additive action of 5FC/FLC not only in $f c y 2$ mutants of $S$. cerevisiae but also in a furl strain, which is fully capable of 5FC import, we suggest that aided 5FC penetration by membranedamaging antimycotics may increase synergism in 5FCresistant strains, but is probably not generally required.

\section{Effects of 5FC and FLC on spontaneously occurring resistant clones}

To elucidate whether residual antimycotic response in spontaneous mutants is indeed detectable and functionally linked with susceptibility to combined 5FC/FLC application, naturally occurring drug-resistant mutants in $S$. cerevisiae were screened. Among 73 clones obtained in a screening for 5FC tolerance, 39 turned out to be stable. Almost all of the latter displayed additive effects when treated jointly with both drugs; only three of them eventually turned out to be fully resistant, and interestingly, for these isolates, the 5FC/FLC combination had the same effect as the singly applied 5FC (Table 3). All strains obtained in a screening for FLC tolerance $(n=36)$ still displayed residual drug sensitivity. Remarkably, combined application of both antimycotic compounds increased their biological activities in either case, supporting the notion 
(a)

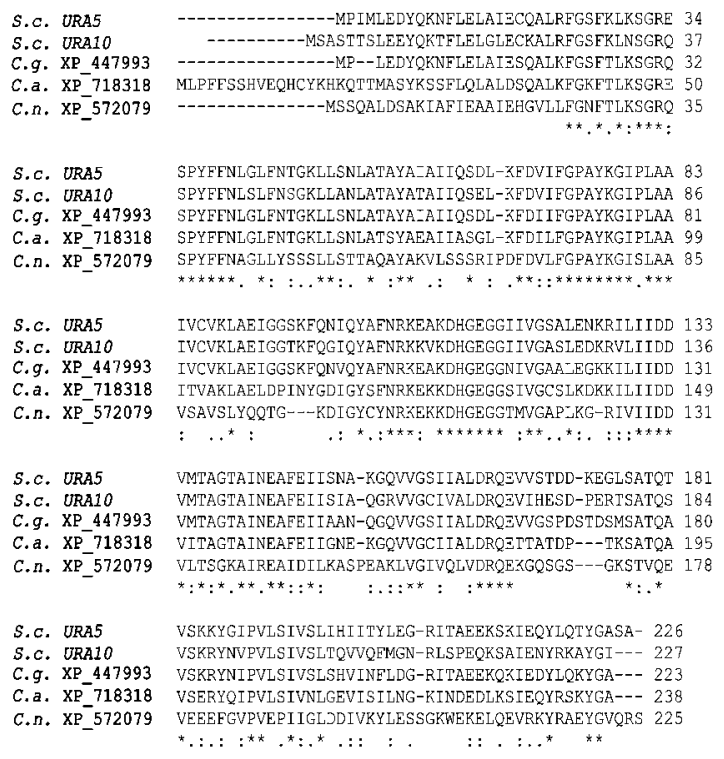

(b)

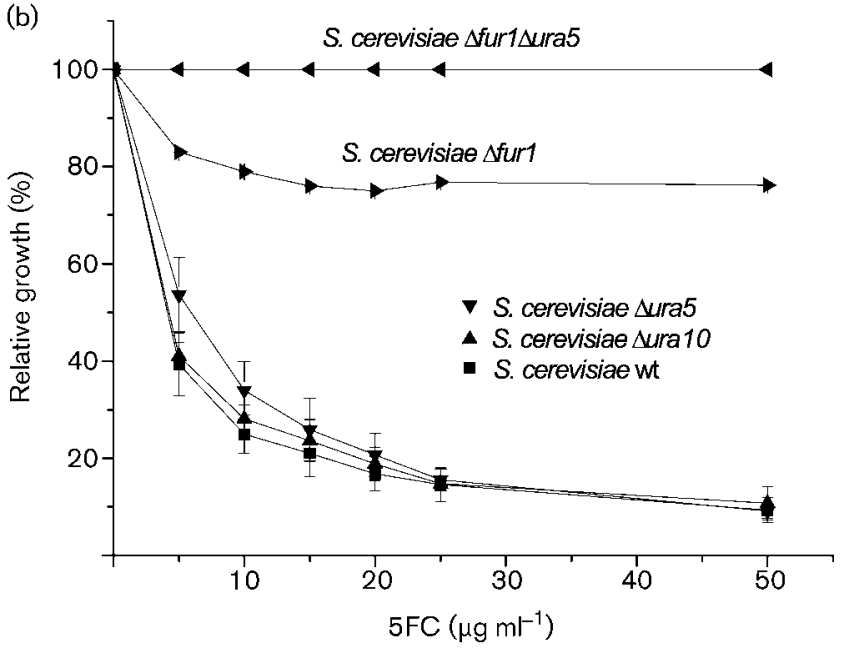

Fig. 5. (a) Amino acid alignment of orotate phosphoribosyl transferases (OPRTs), comparing S. cerevisiae Ura5 and Ura10 sequences with Candida glabrata (XP_447993), Candida albicans (XP_718318) and Cryptococcus neoformans (XP_572079); only one ORPT exists in the latter three species. All OPRT accession numbers refer to those deposited in the NCBI database. (b) Growth of $\Delta u r a 5, \Delta u r a 10$ and $\Delta$ fur 1 single mutants, and a $\Delta$ ura5 $\Delta$ fur 1 double mutant, with 5FC. 5FC concentrations ranging from 0 to $50 \mu \mathrm{g} \mathrm{ml}^{-1}$ were tested and relative growth was measured as for Fig. 3. Error bars represent SD (not plotted where smaller than symbols).

that residual drug response is required and sufficient for additive antifungal activity of the 5FC/FLC combination (Table 3). It is noteworthy that occurrence of full resistance to either drug was an exception rather than the rule in our experiments. In fact we have experienced it only for the three strains being fully resistant to 5FC. In all other
Table 3. Screening for spontaneous mutants to $5 F C$ and FLC resistance

A cell suspension of (S. cerevisiae BY4741) was plated out on YPD plates containing 5FC $\left(100 \mu \mathrm{g} \mathrm{ml}^{-1}\right)$ or FLC $\left(100 \mu \mathrm{g} \mathrm{ml}^{-1}\right)$ and incubated at $30{ }^{\circ} \mathrm{C}$ for approximately 5 days. Colony-forming units capable of growing in the presence of each agent were cultivated further for 1 day under the same conditions. Those that showed stable resistance were additionally tested by the micotitre plate assay at concentrations ranging from 10 to $100 \mu \mathrm{g} \mathrm{ml}^{-1}$ of each agent. Those strains classified as partially resistant displayed a relative growth of at least $25 \%$ above the wild-type strain at concentrations up to $25 \mu \mathrm{g}$ $\mathrm{ml}^{-1}$, while those observed as resistant showed no response at all to the concentrations of 5FC and FLC applied.

\begin{tabular}{|lccc|}
\hline $\begin{array}{l}\text { Antimycotic } \\
\text { compound }\end{array}$ & $\begin{array}{c}\text { No. } \\
\text { obtained }\end{array}$ & $\begin{array}{c}\text { No. fully } \\
\text { resistant/ } \\
\text { additivity } \\
\text { detected }\end{array}$ & $\begin{array}{c}\text { No. partially } \\
\text { resistant/ } \\
\text { additivity } \\
\text { detected }\end{array}$ \\
\hline 5FC & 73 & $3 / 0$ & $36 / 36$ \\
FLC & 36 & $0 / 0$ & $36 / 36$ \\
\hline
\end{tabular}

instances susceptibility to combined drug treatment was prevalent.

Since full FLC resistance requires a simultaneous loss of function of Erg3 and Erg11, whereas 5FC resistance is brought about by a mutation in a single gene (FCY1) our screening data fit with the drug sensitivity profiles for defined mutants (see above), as functional disturbances of 5FC uptake and metabolism can be bypassed by alternative permeases (Paluszynski et al., 2006) or phosphoribosyltransferases (Fig. 5b), respectively. The finding that primary resistance does not generally abolish the effectiveness of 5FC, in particular when combined with FLC, may support the use of this agent in clinical applications despite the known rapid establishment of spontaneous resistance. Clearly, however, when 5FC resistance is due to loss of function of the cytosine deaminase, 5FC application is not appropriate.

\section{Testing combined drug efficiency in defined mutants with primary resistance to 5FC and FLC}

Double mutants carrying mutations conferring resistance to both agents were generated to check whether partial resistance to either 5FC or FLC still allows efficient combined drug action (Fig. 6). Indeed, combination of the $\Delta e r g 3$ mutation with either $\Delta f c y 2$ or $\Delta f u r 1$ resulted in strains displaying robust resistance to both 5FC and FLC. However, as for the respective single mutants, residual response to both 5FC and FLC was observed in the $\Delta \operatorname{erg} 3$ $\Delta f c y 2(\mathrm{FIC}=0.57)$ and the $\Delta$ erg3 $\Delta$ furl strain, where a distinct FIC could not be determined (Fig. 6a, b). Combined application of both drugs led to increased antifungal activity; thus even a primary resistance against two antifungal agents does not a priori exclude additive 
(a) $\Delta f c y 1 \Delta e r g 3$

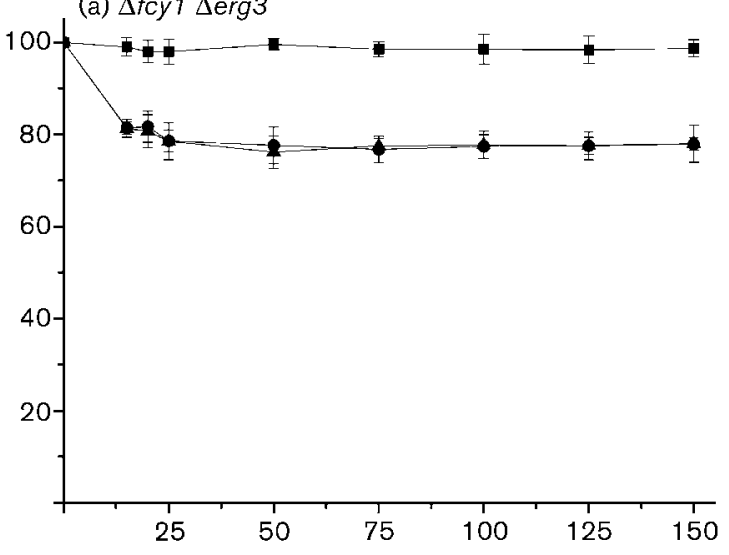

(b) $\Delta$ tcy $2 \Delta e r g 3$

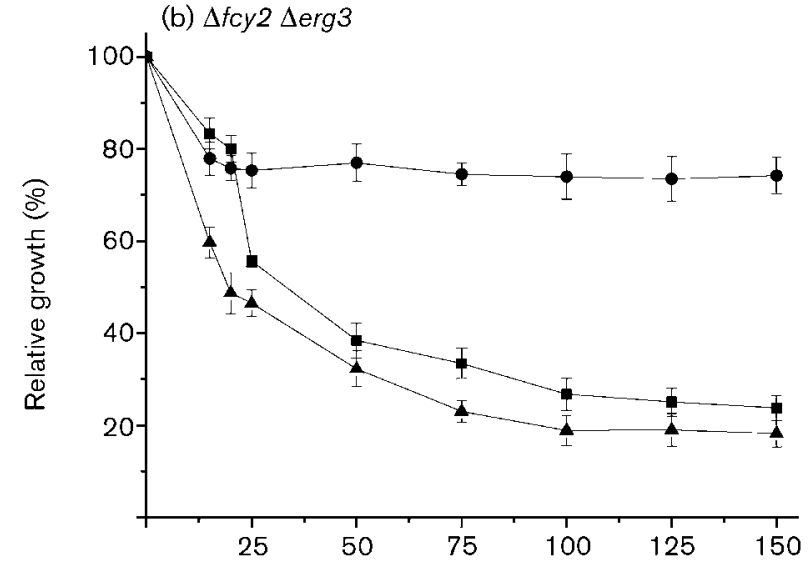

(c) $\Delta$ furt $\Delta$ erg 3

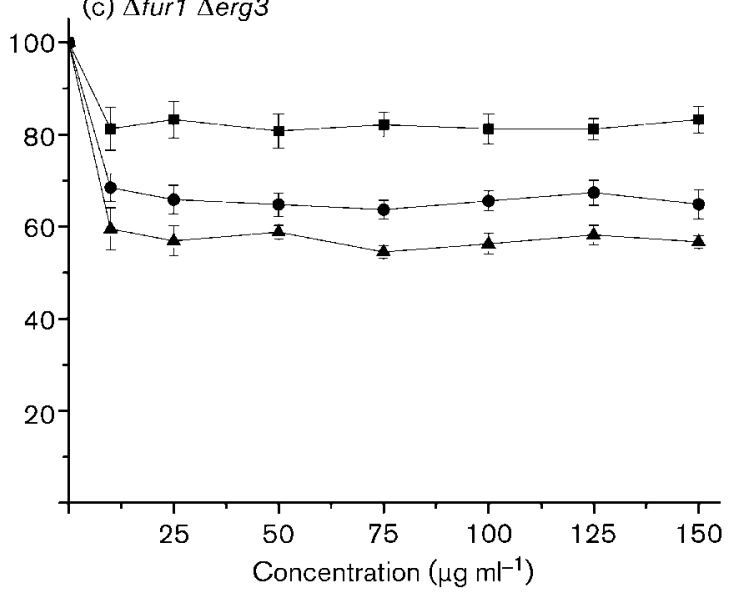

Fig. 6. Characterization of double mutant strains, carrying defects in both $5 \mathrm{FC}$ and ergosterol metabolic pathways, for $5 \mathrm{FC} / \mathrm{FLC}$ additivity. (a) $\Delta f c y 1 \Delta e r g 3,($ b) $\Delta f c y 2 \Delta e r g 3$, and (c) $\Delta f u r 1 \Delta e r g 3$ treated with $5 \mathrm{FC}(\boldsymbol{\square}), \mathrm{FLC}(\mathbf{0})$ and $5 \mathrm{FC}+\mathrm{FLC}(\mathbf{\Delta})$. Microtitre plate tests were carried out and relative growth was calculated as for Fig. 3. Error bars represent SD (not plotted where smaller than symbols). effects in combinational treatments. Combining full 5FC $(\Delta f c y 1)$ with partial FLC ( $\Delta \operatorname{erg} 3)$ resistance, however, completely abolished additivity, supporting the conclusion that at least a faint residual response to both of the drugs is required (Fig. 6a).

\section{Conclusions}

The majority of mutations leading to tolerance to 5FC and FLC affect genes that confer only partial resistance against those compounds. Only in rather rare cases is full resistance established and combined drug treatment not effective; in all other instances antifungal treatment by 5FC/FLC in combination is feasible. Careful examination of drug responses in clinical isolates is thus necessary, as resistance against one or even both of the agents does not a priori exclude an efficient therapy by combined application of 5FC and FLC.

\section{ACKNOWLEDGEMENTS}

Thanks are due to Dr M. Bard for providing yeast strains and Dr M. Rohe for providing antifungal agents.

\section{REFERENCES}

Agarwal, A. K., Rogers, P. D., Baerson, S. R., Jacob, M. R., Barker, K. S., Cleary, J. D., Walker, L. A., Nagle, D. G. \& Clark, A. M. (2003). Genome-wide expression profiling of the response to polyene, pyrimidine, azole, and echinocandin antifungal agents in Saccharomyces cerevisiae. J Biol Chem 278, 34998-35015.

Alexander, B. D. \& Perfect, J. R. (1997). Antifungal resistant trends towards the year 2000: Implications of therapy and new approaches. Drugs 54, 657-678.

Allendoerfer, R., Marquis, A. J., Rinaldi, M. J. \& Graybill, J. R. (1991). Combined therapy with fluconazole and flucytosine in murine cryptococcal meningitis. Antimicrob Agents Chemother 35, 726-729.

Arthington, B. A., Bennett, L. G., Skatrud, P. L., Guynn, C. J., Barbuch, R. J., Ulbright, C. E. \& Bard, M. (1991). Cloning, disruption, and sequence of the gene encoding yeast C-5 sterol desaturase. Gene 102, $39-44$.

Bammert, G. F. \& Fostel, J. M. (2000). Genome-wide expression patterns in Saccharomyces cerevisiae: comparison of drug treatments and genetic alterations affecting biosynthesis of ergosterol. Antimicrob Agents Chemother 44, 1255-1265.

Bard, M., Lees, N. D., Turi, T., Craft, D., Cofrin, L., Barbuch, R., Koegel, C. \& Loper, J. C. (1993). Sterol synthesis and viability of erg11 (cytochrome P450 lanosterol demethylase) mutations in Saccharomyces cerevisiae and Candida albicans. Lipids 28, 963-967.

Barns, S. M., Lane, D. J., Sogin, M. L., Bibeau, C. \& Weisburg, W. G. (1991). Evolutionary relationships among pathogenic Candida species and relatives. J Bacteriol 173, 2250-2255.

Chevallier, M. R., Jund, R. \& Lacroute, F. (1975). Characterization of cytosine permeation in Saccharomyces cerevisiae. J Bacteriol 122, 629-641.

de Kruijff, B. \& Demel, R. A. (1974). Polyene antibiotic-sterol interactions in membranes of acholeplasma-laidlawii cells and lecithin liposomes. 3. Molecular structure of polyene antibiotic-cholesterol complexes. Biochim Biophys Acta 339, 57-70. 
de Kruijff, B., Gerritsen, W. J., Oerleman, A., Demel, R. A. \& Van Deenen, L. L. M. (1974). Polyene antibiotic-sterol interactions in membranes of Acholeplasma laidlawii cells and lecithin liposomes. 1. Specificity of membrane-permeability changes induced by polyene antibiotics. Biochim Biophys Acta 339, 30-43.

de Montigny, J., Kern, L., Hubert, J. C. \& Lacroute, F. (1990). Cloning and sequencing of URA10, a second gene encoding orotate phosphoribosyl transferase in Saccharomyces cerevisiae. Curr Genet 17, 105-111.

Elfopouios, G. M. \& Moellering, R. C., Jr (1991). Antimicrobial combinations. In Antibiotics in Laboratory Medicine, 3rd edn, pp. 432-492. Edited by U. Lorian. Baltimore, MD: Williams \& Wilkins.

Erbs, P., Exinger, F. \& Jund, R. (1997). Characterization of the Saccharomyces cerevisiae FCY1 gene encoding cytosine deaminase and its homologue FCA1 of Candida albicans. Curr Genet 31, 1-6.

Fasoli, M. O., Kerridge, D., Morris, P. G. \& Torosantucci, A. (1990). ${ }^{19} \mathrm{~F}$ nuclear magnetic resonance study of fluoropyrimidine metabolism in strains of Candida glabrata with specific defects in pyrimidine metabolism. Antimicrob Agents Chemother 34, 1996-2006.

Ghannoum, M. A. \& Rice, L. B. (1999). Antifungal agents mode of action, mechanisms of resistance, and correlation of these mechanisms with bacterial resistance. Clin Microbiol Rev 12, 501-517.

Gietz, R. D. \& Schiestl, R. H. (1995). Transforming yeast with DNA. Methods Mol Cell Biol 5, 255-269.

Gueldener, U., Heinisch, J., Koehler, G. J., Voss, D. \& Hegemann, J. H. (2002). A second set of loxP marker cassettes for Cre-mediated multiple gene knockouts in budding yeast. Nucleic Acids Res 30, e23.

Hartmann, K. U. \& Heidelberger, C. (1961). Studies on fluorinated pyrimidines. XIII. Inhibition of thymidylate synthetase. J Biol Chem 236, 3006-3013.

Hendriks, L., Goris, A., Neefs, J., van de Peer, Y., Hiennebert, G. \& de Wachter, R. (1989). The nucleotide sequence of the small ribosomal subunit RNA of the yeast Candida albicans and the evolutionary position of the fungi among the eukaryotes. Syst Appl Microbiol 12, 223-229.

Hitchcock, C. A. (1991). Cytochrome P-450-dependent $14 \alpha$-sterol demethylase of Candida albicans and its interaction with azole antifungals. Biochem Soc Trans 19, 782-787.

Johnson, D. M., MacDougall, C., Ostrosky-Zeichner, L., Perfect, J. R. \& Rex, J. R. (2004). Combination antifungal therapy. Antimicrob Agents Chemother 48, 693-715.

Joseph-Horne, T. \& Hollomon, D. W. (1997). Molecular mechanisms of azole resistance in fungi. FEMS Microbiol Lett 149, 141-149.

Jund, R. \& Lacroute, F. (1970). Genetic and physiological aspects of resistance to 5-fluoropyrimidines in Saccharomyces cerevisiae. J Bacteriol 102, 607-615.

Kaiser, C., Michaelis, S. \& Mitchell, A. (1994). Methods in Yeast Genetics. Cold Spring Harbor, NY: Cold Spring Harbor Laboratory.

Kalb, V. F., Woods, C. W., Turi, T. G., Dey, C. R., Sutter, T. R. \& Loper, J. C. (1987). Primary structure of the P450 lanosterol demethylase gene from Saccharomyces cerevisiae. DNA 6, 529-537.

Kelly, S. L., Lamb, D. C., Corran, A. J., Baldwin, B. C. \& Kelly, D. E. (1995). Mode of action and resistance to azole antifungals associated with the formation of 14 alpha-methylergosta-8,24(28)-dien-3beta,6-alpha-diol. Biochem Biophys Res Commun 207, 910-915.

Kern, L., de Montigny, J., Jund, J. \& Lacroute, F. (1990). The FUR1 gene of Saccharomyces cerevisiae: cloning, structure and expression of wild-type and mutant alleles. Gene 88, 149-157.

Kurtz, J. E., Exinger, F., Erbs, P. \& Jund, R. (1999). New insights into the pyrimidine salvage pathway of Saccharomyces cerevisiae: requirement of six genes for cytidine metabolism. Curr Genet 36, 130-136.
Lupetti, A., Danesi, R., Campa, M., Del Tacca, M. \& Kelly, S. (2002). Molecular basis of resistance to azole antifungals. Trends Mol Med $\mathbf{8}$, $76-81$.

Mukherjee, P. K., Sheehan, D. J., Hitchcock, C. A. \& Ghannoum, M. A. (2005). Combination treatment of invasive fungal infections. Clin Microbiol Rev 18, 163-194.

NCCLS (1998). Reference method for broth dilution antifungal susceptibility testing of conidium-forming filamentous fungi: proposed standard M38-P. Wayne, PA: National Committee for Clinical Laboratory Standards.

Paluszynski, J. P., Klassen, R., Rohe, M. \& Meinhardt, F. (2006). Various cytosine/adenine permease homologues are involved in the toxicity of 5-fluorocytosine in Saccharomyces cerevisiae. Yeast 23, 707-715.

Peters, G. J., Laurensse, E., Lankelma, J., Leyva, A. \& Pinedo, H. M. (1984). Separation of several 5-fluorouracil metabolites in various melanoma cell lines. Evidence for the synthesis of 5-fluorouracil nucleotide sugars. Eur J Cancer Clin Oncol 20, 1425-1431.

Polak, A. \& Scholer, H. (1975). Mode of action of 5-fluorocytosine and mechanisms of resistance. Chemotherapy 21, 113-130.

Sambrook, J., Fritsch, E. F. \& Maniatis, T. (1989). Molecular Cloning: a Laboratory Manual, 2nd edn. Cold Harbor, NY: Cold Spring Harbor Laboratory.

Sanglard, D., Ischer, F., Parkinson, T., Falconer, D. \& Bille, J. (2003). Candida albicans mutations in the ergosterol biosynthesis pathway and resistance to several other agents. Antimicrob Agents Chemother 47, 2404-2412.

Schwarz, P., Dromer, F., Lortholary, O. \& Dannaoui, E. (2003). In vitro interaction of flucytosine with conventional and new antifungals against Cryptococcus neoformans clinical isolates. Antimicrob Agents Chemother 47, 3361-3364.

Schwarz, P., Dromer, F., Lortholary, O. \& Dannaoui, E. (2006). Efficacy of amphotericin B in combination with flucytosine against flucytosine-susceptible or flucytosine-resistant isolates of Cryptococcus neoformans during disseminated murine cryptococcosis. Antimicrob Agents Chemother 50, 113-120.

Schwarz, P., Janbon, G., Dromer, F., Lortholary, O. \& Dannaoui, E. (2007). Combination of amphotericin B with flucytosine is active in vitro against flucytosine-resistant isolates of Cryptococcus neoformans. Antimicrob Agents Chemother 51, 383-385.

Smith, S. J., Crowley, J. H. \& Parks, L. W. (1996). Transcriptional regulation by ergosterol in the yeast Saccharomyces cerevisiae. Mol Cell Biol 16, 5427-5432.

Southern, E. M. (1975). Detection of specific sequences among DNA fragments separated by gel electrophoresis. J Mol Biol 98, 503-517.

Te Dorsthorst, D. T. A., Verweij, P. E., Meletiadis, J., Bergervoet, M., Punt, N. C., Meis, J. F. G. M. \& Mouton, J. W. (2002). In vitro interaction of flucytosine combined with amphotericin $B$ or fluconazole against thirty-five yeast isolates determined by both the fractional inhibitory concentration index and the response surface approach. Antimicrob Agents Chemother 46, 2982-2989.

Vanden Bossche, H. (1985). Biochemical targets for antifungal azole derivatives: hypothesis on the mode of action. In Current Topics in Medical Mycology, vol. 1, pp. 313-351. Edited by M. R. McGinnis. New York: Springer-Verlag.

Vanden Bossche, H., Willemsens, G. \& Marichal, P. (1987). AntiCandida drugs: the biochemical analysis for their activity. Crit Rev Microbiol 15, 57-72.

Vanden Bossche, H., Marichal, P., Odds, F. C., Jeune, L. \& Coene, M. C. (1992). Characterization of an azole-resistant Candida glabrata isolate. Antimicrob Agents Chemother 36, 2602-2610. 
Vanden Bossche, H., Marichal, P. \& Odds, F. C. (1994). Molecular mechanisms of drug resistance in fungi. Trends Microbiol 2, 393-400.

Wach, A., Brachat, A., Pöhlmann, R. \& Philippsen, P. (1994). New heterologous modules for classical or PCR-based gene disruptions in Saccharomyces cerevisiae. Yeast 10, 1793-1808.

Wadler, S., Horowitz, R., Zhang, H. Y. \& Schwartz, E. L. (1998). Effects of perturbations of pools of deoxyribonucleoside triphosphates on expression of ribonucleotide reductase, a G1/S transition state enzyme, in p53-mutated cells. Biochem Pharmacol 55, 1353-1360.

Watson, P. F., Rose, M. E. \& Kelly, S. L. (1988). Isolation and analysis of ketoconazole mutants of Saccharomyces cerevisiae. J Med Vet Mycol 26, 153-162.
Watson, P. F., Rose, M. E., Ellis, S. W., England, H. \& Kelly, S. L. (1989). Defective sterol C5-6 desaturation and azole resistance: a new hypothesis for the mode of action of azole anti-fungals. Biochem Biophys Res Commun 164, 1170-1175.

Whelan, W. L. (1987). The genetic basis of resistance to 5fluorocytosine in Candida species and Cryptococcus neoformans. Crit Rev Microbiol 15, 45-56.

Yanisch-Perron, C., Viera, J. \& Messing, J. (1985). Improved M13 phage cloning vectors and host strains: nucleotide sequence of the M13mp18 and pUC19 vectors. Gene 33, 103-119.

Edited by: J. Pla 